\title{
L'intertextualité ravageuse de Bouvard et Pécuchet
}

\section{Anne Herschberg Pierrot et Jacques Neefs}

\section{CpenEdition}

\section{Journals}

Édition électronique

URL : https://journals.openedition.org/genesis/5686

DOI : 10.4000/genesis.5686

ISSN : 2268-1590

\section{Éditeur :}

Presses universitaires de Paris Sorbonne (PUPS), Société internationale de génétique artistique littéraire et scientifique (SIGALES)

\section{Édition imprimée}

Date de publication : 15 décembre 2020

Pagination : 85-100

ISBN : 979-10-231-0704-3

ISSN : 1167-5101

\section{Référence électronique}

Anne Herschberg Pierrot et Jacques Neefs, «L'intertextualité ravageuse de Bouvard et Pécuchet », Genesis [En ligne], 51 | 2020, mis en ligne le 20 décembre 2021, consulté le 11 janvier 2023. URL : http://journals.openedition.org/genesis/5686 ; DOI : https://doi.org/10.4000/genesis.5686 


\title{
L'intertextualité ravageuse de Bouvard et Pécuchet
}

\author{
Anne Herschberg Pierrot et Jacques Neefs
}

L 'intertextualité que Bouvard et Pécuchet mobilise est tout à fait singulière. Comme La Tentation de saint Antoine dont il présente la «contrepartie moderne», Bouvard et Pécuchet est un livre «où se joue la fiction des livres» (M. Foucault). Mais, à la différence de La Tentation, dans Bouvard et Pécuchet les livres participent d'un double jeu. Ils sont lus par Flaubert pour écrire en deux «volumes» ce qu'il appelle «une espèce d'encyclopédie critique en farce ${ }^{1}$ ». Le premier volume, c'est-à-dire les dix premiers chapitres, est consacré à la quête encyclopédique des deux «bonshommes». Puis, après leurs échecs successifs, et avec l'hostilité grandissante du village de Chavignolles contre ces deux « originaux», vient la «bonne idée » finale, celle de la «Copie» du «second volume» (les chapitres XI et XII). Ce projet, laissé en suspens à la mort de Flaubert, est décrit dans les scénarios, et en grande partie mis en œuvre dans le «chantier» que constituent les dossiers de Bouvard et Pécuchet ${ }^{2}$. Le «second volume» devait faire reparaitre des extraits des notes prises sur les livres précédemment lus par Flaubert, et par Bouvard et Pécuchet pour leurs recherches, que les personnages copient et tentent de classer, fabriquant des parallèles et des contradictions entre les opinions, pour finir sur la décision de simplement «copier», indifféremment.

Flaubert insiste à plusieurs reprises sur la nécessité de comprendre son œuvre comme un ensemble. «Ce sacré bouquin me fait vivre dans le tremblement! Il n'aura de signification que par son ensemble» écrit-il le 5 octobre 1877 à Émile Zola. L'entrecroisement génétique et intertextuel des deux volumes est particulièrement complexe. Les deux parties entretiennent une dimension génétique commune. Les aventures et les débats encyclopédiques du roman sont conçus à partir des notes de lecture relevées par Flaubert dans les très nombreux ouvrages qui constituent la bibliothèque génétique de référence de l'œuvre ${ }^{3}$. Mais des extraits de ces notes sont également destinés à être projetés, montrés, et montés dans la «Copie », c'est-à-dire l'ensemble des citations distribuées et classées qui devait paraître avec d'autres documents manuscrits et imprimés ${ }^{4}$ - et en proximité avec le Dictionnaire des idées reçues, le Catalogue des idées chic et d'autres «morceaux fictifs». Il s'établit alors une circulation intertextuelle spécifique entre les deux parties du roman qui entrecroisent les références, et un singulier brouillage entre le travail d'exogenèse et une pratique d'endogenèse 5 .

L'intertextualité mise en jeu requiert une définition large et restreinte tout à la fois. Il y va d'une interdiscursivité généralisée des discours (au sens de Barthes et Kristeva) et d'une intertextualité restreinte plus «textuelle», «littéraire» voire littérale, qui implique la citation, l'allusion (Genette) et la référence. Flaubert construit un dispositif à la fois sérieux et malicieux, ravageur contre les arrogances, dont nous évoquerons trois aspects : la condensation, la fiction, la contrebande.

1. Dans une lettre à Edma Roger des Genettes, le 19 août 1872.

2. Les dossiers pour Bouvard et Pécuchet sont conservés pour l'essentiel à la Bibliothèque municipale de Rouen sous la cote gg 10 («Plans »), g 224 (manuscrit autographe), g 225 (9 volumes de brouillons) et g 226 (dossiers pour la Copie et notes de lecture). Les plans, scénarios et brouillons sont édités en ligne sur le site Flaubert de l'université de Rouen, sous la direction d'Yvan Leclerc (flaubert.univ-rouen.fr). Les dossiers de notes de lecture ont fait l'objet d'une édition en ligne sous la direction de Stéphanie Dord-Crouslé (dossiers-flaubert.fr).

3. «Savez-vous à combien se montent les volumes qu'il m'a fallu absorber pour mes deux bonshommes ? - À plus de 1500 ! [...] Mais cette surabondance de documents m'a permis de n'être pas pédant. De cela, j'en suis sûr» (24 [25] janvier 1880, à Edma Roger des Genettes).

4. Ce devait être «comme le retournement d'une fiction déjà déployée», écrivent Claude Mouchard et Jacques Neefs, dans «Vers le second volume : Bouvard et Pécuchet », Flaubert à l'œuvre, Flammarion, 1980, p. 180 .

5. Sur cette question, voir aussi Anne Herschberg Pierrot, Claude Mouchard, Jacques Neefs, «Les bibliothèques de Flaubert», dans P. d'Iorio et D. Ferrer (dir.), Bibliothèques d'écrivains, CNRS Éditions, 2001, p. 121-144. 


\section{Condensé, concentré}

Céard, à la parution en 1881 du roman de Flaubert, est profondément heurté par l'indécision que produit l'œuvre, et l'incertitude de son «genre»: «L'œuvre est purement philosophique. Rien ne ressemble moins à un roman. » En fait, il lit bien le livre, louant la rigueur et la densité de sa prose : «[Flaubert] avait réduit son écriture au strict nécessaire, économisant les mots, condensant les effets, résumant les sensations, s'évertuant à faire de l'œuvre d'art quelque chose de suggestif, quelque chose de substantiel, qui donne l'idée d'une littérature Liebig6.» «Littérature Liebig»? La figure est étrange : littérature «concentrée» comme les «extraits de viande» inventés par le chimiste agronome ${ }^{7}$ ? De fait, la prose du roman est un formidable concentré de citations, de matières intellectuelles, de propositions résumées, de théories abrégées, mises en mouvement sous forme d'arguments, de résumés, d'échanges, de dialogues directs. Le texte produit de l'incertitude à chaque étape du parcours encyclopédique, en broyant les textes lus puis cités, utilisés en trame du récit ou en citations piratées. Mais Céard lit comme à l'envers la portée du roman, quand il rejette ce qui donne à celui-ci sa force et son exceptionnelle identité, et relève «la grande inquiétude intellectuelle qui en résulte. Il laisse le lecteur indécis et l'esprit en suspens.»

Le trouble est puissant, en effet, qui ressort de la densité des références qui affleurent et de ce que l'on perçoit comme un substrat complexe de «savoirs», de «pensées», d' «hypothèses » et $\mathrm{d}$ ' «opinions », captées dans les bibliothèques les plus diverses, puis rassemblées et condensées, pour faire débat comique. Nous donnons quelques exemples de ces condensations.

\section{Chimie}

Ayant échoué dans l'agriculture, les deux bonshommes concluent: "C'est que peut-être nous ne savons pas la chimie 8 ! » La riposte est immédiate, c'est le début du chapitre III, celui des sciences :

Pour savoir la chimie, ils se procurèrent le cours de Regnault - et apprirent d'abord que «les corps simples sont peut-être composés ».

On les distingue en métalloïdes et en métaux, - différence qui n'a «rien d'absolu», dit l'auteur. De même pour les acides et les bases, «un corps pouvant se comporter à la manière des acides ou des bases, suivant les circonstances».

La notation leur parut baroque. Les Proportions multiples troublèrent Pécuchet.

- «Puisqu'une molécule de A, je suppose, se combine avec plusieurs parties de $\mathrm{B}$, il me semble que cette molécule doit se diviser en autant de parties. Mais si elle se divise, elle cesse d'être l'unité, la molécule primordiale. Enfin, je ne comprends pas.»

- «Moi, non plus!» disait Bouvard.

Et ils recoururent à un ouvrage moins difficile, celui de Girardin - où ils acquirent la certitude que dix litres d'air pèsent cent grammes, qu'il n'entre pas de plomb dans les crayons, que le diamant n'est que du carbone. (g 224, fo 47) ${ }^{9}$

Le recours au Cours élémentaire de Chimie (1847-1849) de Regnault, professeur au Collège de France et à Polytechnique, est d'actualité par rapport à la fiction ${ }^{10}$, et il a valeur d'autorité car il représente un développement nouveau de la chimie expérimentale et analytique, autour de la notion de composition atomique. Mais Flaubert condense ici en quelques paragraphes ce que Regnault présente comme une hypothèse sur l'avenir de la chimie :

Il est très possible que les progrès futurs de la science nous permettent, par la suite, d'opérer des décompositions qui ont résisté à nos moyens actuels; et qu'alors un certain nombre des corps que nous regardons aujourd'hui comme simples, peut-être même tous ces corps, seront considérés comme des corps composés 11 .

\section{L'Express, 9 avril 1881.}

7. Liebig est mentionné dans les notes de Flaubert sur la chimie et l'agronomie.

8. Fin du ch. II (g 224, fo 46), GF, p. 106; LP, p. 96. L'exclamation est pertinente quant au développement, sous la monarchie de Juillet, d'une agriculture scientifique moderne, comme l'a montré Jean Gayon, "Agriculture et agronomie dans Bouvard et Pécuchet de Gustave Flaubert», Littérature, no 109, mars 1988. Les références à Bouvard et Pécuchet renvoient à l'édition Garnier Flammarion (GF) par Stéphanie Dord-Crouslé, 1999, et à l'édition du Livre de Poche (LP) par Pierre-Marc de Biasi, 1999.

9. GF, p. 107; LP, p. 96.

10. Avec cependant une certaine «avance» vers l'avenir si l'on date l'épisode agricole, comme le fait Dumesnil, à la suite de Descharmes, de 1841 à 1844 ( «Plan chronologique de Bouvard et Pécuchet», Bouvard et Pécuchet, Les Belles Lettres, 1945, t. II, p. 368-369).

11. M. V. Regnault, Cours élémentaires de chimie, Masson, Langlois et Leclercq, 4e éd., 1854, p. 3. 
De même, en ce qui concerne «la loi des proportions multiples», Flaubert prête à ses personnages une incompréhension qui repose sur une logique simple de «molécule primordiale», alors que précisément le développement de la chimie qu'engage Regnault établit la loi des proportions multiples pour, non pas partir de l'idée de molécule primordiale, mais au contraire «établir la divisibilité limitée de la matière et l'existence des molécules indivisibles 12 », ce qui sera pour longtemps le cours de la chimie moderne. Flaubert concentre de fait une révolution disciplinaire en cours - et un long traité «moderne» de chimie en quatre volumes - en ces quelques formules de perplexité, qui ont éventuellement pu être rédigées à partir de la lecture de la seule introduction du Cours. Nous ne disposons pas des notes prises par Flaubert. Mais celui-ci prête aux deux personnages sa propre incompréhension : «Je lis maintenant de la chimie (à laquelle je ne comprends goutte)» (à George Sand, 3 février 1873). Il peut du moins mettre en prose l'objet et les dimensions épistémologiques de cette «incompréhension». Et l'on peut également imaginer que ce paradigme «chimique» de la décomposition et des recompositions des molécules a une puissance figurale toute particulière pour un livre qui se compose de la fragmentation des textes, en extraits recomposés pour un tout autre propos que leur propos original ${ }^{13}$. Enfin, le recours au manuel de Jean Girardin, Leçons de chimie élémentaire appliquée aux arts industriels et faites le dimanche à l'école municipale de Rouen (1re éd. 1836), est une parfaite référence de vulgarisation alors déjà ancienne, et rouennaise, qui permet de présenter bord à bord deux états bien différents de la discipline. Le discours du roman conquiert une capacité singulière de concentration et d'interdiscursivité épistémologique, fixant en aventures de pensées, d'interprétations, de mésententes, une matière scientifique complexe et mobile.

\section{Déluge}

Il s'agit pour Flaubert de rendre les savoirs, les discours, les livres, les textes qu'il convoque disponibles pour des scénographies comiques susceptibles d'exposer de manière synthétique de profonds conflits d'interprétation sous forme de ce qu'il nomme un «comique d'idées ${ }^{14}$ ».

Ayant abandonné, à la suite d'une catastrophique expédition «géologique» et après la lecture d'un feuilleton (fictif et trouvé par hasard) qui décrit les théories nouvelles sur la formation de la terre, le prestige des théories de Cuvier, Bouvard et Pécuchet se tournent vers de nouvelles lectures :

Par des biographies et des extraits, ils apprirent quelque chose des doctrines de Lamarck et de Geoffroy Saint-Hilaire.

Tout cela contrariait les idées reçues, l'autorité de l'Église.

Bouvard en éprouva comme l'allègement d'un joug brisé.

- «Je voudrais voir, maintenant, ce que le citoyen Jeufroy me répondrait sur le Déluge !» 15

Le déluge mosaïque est encore, dans les années 1840, l'objet de vives controverses. Le débat entre Bouvard et l'abbé Jeufroy est longuement préparé dans une série de brouillons. On peut lire dans l'un d'eux, parmi d'autres 16, comment Flaubert articule argument et références :

Perdent la foi en Cuvier relativement aux Créations Successives (Boué)

Cherchent le curé et le provoquent devant témoins sur la question du Déluge (Buffon, Voltaire, Laroque)

Le curé leur répond par un argument qui les embarrasse.

Les animaux trouvés dans la glace sont morts tout à coup

(Pouchet) - alors ils doutent de la géologie

Ils voudraient tenir la philosophie des Sciences.

\section{Ibid., p. 6.}

13. Une autre figure «scientifique», qui fait suite à la chimie moléculaire dans le cours de Regnault, semble informer la logique du traitement des textes engagée par Flaubert, celle de la «cristallographie». On trouve dès les scénarios du Carnet 19, au tout début de la conception de Bouvard et Pécuchet en 1853 : «Pour Les Deux Cloportes / Haüy laissa par maladresse tomber un beau groupe de spath calcaire cristallisé en prismes. Un de ces prismes se brisa de manière à montrer sur sa cassure des faces non moins lisses que celles du dehors et qui présentaient l'apparence d'un cristal nouveau. / Ce hasard mit Haüy sur la voie des découvertes. Il brisa toutes les pièces de sa collection. / Bouvard et Pécuchet l'imitent. Ils brisent tout, espérant trouver un système. Voy. Bibliographie universelle, t. LXVI, p. 532.» (Fo 29, Carnets de travail, éd. P.-M. de Biasi, Balland, 1988, p. 280.)

14. «Je crois qu'on n'a pas encore tenté le comique d'idées. Il est possible que je m'y noie, mais si je m'en tire, le globe terrestre ne sera pas digne de me porter.» (Lettre à Edma Roger des Genettes, 2 avril 1877.)

15. Pour l'ensemble du débat sur le déluge : GF, p. 145-148; LP, p. 140-144 (g 224, fo 73-74).

16. Ms g $225^{3}$, fo 362 (voir fig. 1); pour une rédaction plus avancée voir en particulier g $225^{3}$, fo $401 \mathrm{v}^{\mathrm{o}}$. 
Fig. 1 : Page de brouillon de l'épisode "géologique», ch. III. Ms g 225 ${ }^{3}$, fo 362.

(C) Bibliothèque de Rouen

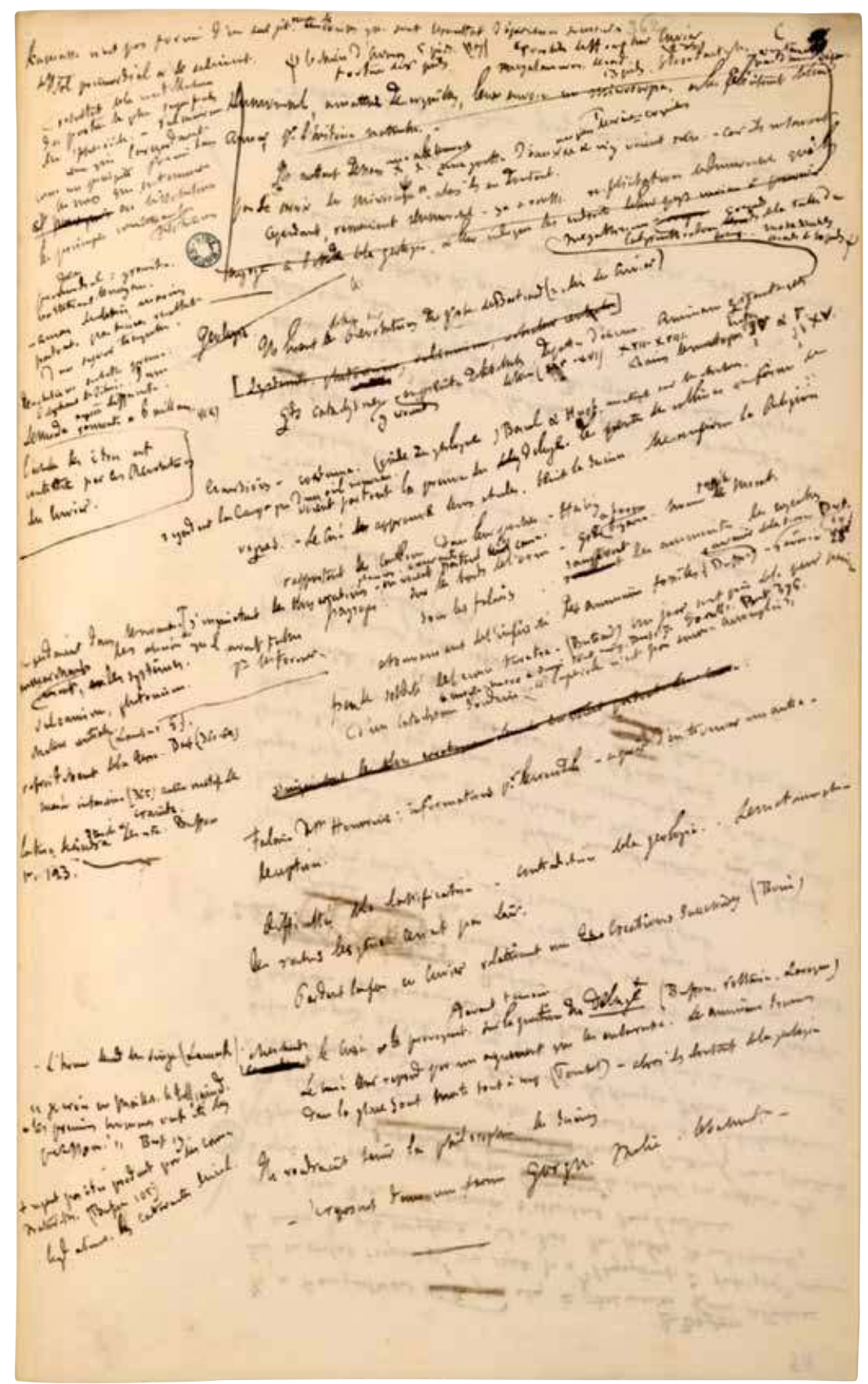

Et en marge :

L'homme descend du singe (Lamarck) «Je crois en Maillet, le Telliamed :

les premiers hommes ont été des poissons! » Bert[rand] 19.

Flaubert référencie et concentre ainsi un débat très complexe qui se déroule sur une longue période. La dévalorisation des théories fixistes et catastrophistes de Cuvier intervient dès les années 1830 avec Lyell en Angleterre (Principles of Geology, 3 vol. 1830, 1832, 1833), puis en France avec sa traduction par Mme T. Meullien (1848), que Flaubert mentionne dans une liste bibliographique sur la géologie (g 2261, fo 243). Lyell s'oppose au catastrophisme de Cuvier par l'idée « uniformitariste» 
d'une transformation lente, continue et toujours contemporaine de la terre. Les débats sur la «géologie» comme discipline spécifique se sont multipliés dans la période de 1830 à 1850 . Les thèses transformistes de Lamarck (transformation des espèces par désuétude et transmission des caractères acquis) et de Geoffroy Saint-Hilaire (unité de composition organique dans une perspective évolutionniste) s'opposaient, elles aussi, à la théorie fixiste de Cuvier. Avec Bouvard et Pécuchet, Flaubert remonte ainsi à un débat épistémologique crucial des années 1830 , et se réfère même à un texte beaucoup plus ancien : Telliamed ou entretiens d'un philosophe indien avec un missionnaire français sur la diminution des mers, de Benoît de Maillet, publié en deux volumes à Amsterdam en 1748. Cette fable dirigée contre le christianisme propose les rudiments d'une théorie transformiste de la terre et des espèces, et imagine l'émersion progressive des montagnes.

En quelques pages de son texte final, Flaubert concentre ainsi une puissante polémique contre la théorie créationniste orthodoxe, par extraction et juxtaposition de références et d'arguments, qui littéralement fondent dans le texte «final», plein pourtant de ce travail secret d'argumentation qui fait la trame référentielle de la scène comique. Jusqu'à l'explosion scandaleuse :

Comme les allées étaient trop petites, en gesticulant, on marchait dans les plates-bandes. Langlois fut pris d'une quinte de toux. Le Capitaine criait : «Vous êtes des révolutionnaires!» Girbal : «La paix ! la paix !» Le prêtre : «Quel matérialisme!» Foureau : «Occupons-nous plutôt de notre chasuble!»

- «Hou ! Laissez-moi parler!» Et Bouvard s'échauffant, alla jusqu'à dire que l'Homme descendait du Singe !

Tous les fabriciens se regardèrent, fort ébahis, et comme pour s'assurer qu'ils n'étaient pas des singes.

Bouvard reprit : - «En comparant le fotus d'une femme, d'une chienne, d'un oiseau, d'une grenouille...»

- «Assez!»

- «Moi, je vais plus loin!» s'écria Pécuchet. «L'Homme descend des poissons! » Des rires éclatèrent. Mais sans se troubler : «le Telliamed! un livre arabe !... » (g 224, fo 75) ${ }^{17}$

Est-ce encore «intertextualité » et «interdiscursivité » quand il s'agit ainsi de concentrer les enjeux fondamentaux d'un débat entre religion, opinion publique et science, rigoureusement même si finalement secrètement référencés?

\section{Hyper philosophie}

Le moment spéculatif du roman, au chapitre VIII, porte les épreuves que Flaubert assigne aux deux bonshommes à leur limite, celle de penser le pensable, d'appréhender le travail philosophique lui-même. L'appropriation intertextuelle et interdiscursive de la pensée et d'une «philosophie » en «comique d'idées » est assurément un palier extrême. La solution adoptée par Flaubert est radicale : porter en récit et en prose ce que peut être la limite de la compréhension.

[Pécuchet] se procura une introduction à la philosophie Hégélienne, et voulut l'expliquer à Bouvard.

- «Tout ce qui est rationnel est réel. Il n’y a même de réel que l'idée. Les lois de l'Esprit sont les lois de l'univers, la raison de l'homme est identique à celle de Dieu.»

Bouvard feignait de comprendre.

- «Donc, l'Absolu c'est à la fois le sujet et l'objet, l'unité où viennent se rejoindre toutes les différences. Ainsi les contradictoires sont résolus. L'ombre permet la lumière, le froid mêlé au chaud produit la température, l'organisme ne se maintient que par la destruction de l'organisme, partout un principe qui divise, un principe qui enchaîne. » (g 224, fo 176) ${ }^{18}$

L'introduction à la philosophie hégélienne dont Flaubert confie à Pécuchet l'exposé combine L'Hégélianisme et la Philosophie et l'Introduction à la philosophie de Hegel d'Augusto Vera, sur lesquels Flaubert a pris des notes ${ }^{19}$. Le «résumé» que fait Pécuchet reprend littéralement des phrases de ces notes ${ }^{20}$. Ce qui est alors frappant est précisément que ce qui «sonne» en effet comme du «Hegel» est rendu étrange, inintelligible. Flaubert accentue encore l'effet dans le débat qu'il ouvre ensuite entre Pécuchet et le curé :

17. GF, p. 148 ; LP, p. 144.

18. GF, p. 300-301; LP, p. 314

19. Ces notes (g 2266, fo 35-36 vo) ont été commentées et publiées par Atsushi Yamazaki dans «Le dossier "philosophie" de Bouvard et Pécuchet. Hegel et Spinoza», Gustave Flaubert, no 6, «Fiction et philosophie», Caen, Lettres Modernes, Minard, 2008, p. 253.

20. Flaubert avait lu les premiers volumes de la traduction-adaptation par Charles Bénard du Cours d'esthétique de Hegel dans les années 1840. Il en reprend la lecture et lit les volumes suivants au moment de la reprise de La Tentation de saint Antoine et de la préparation de Bouvard et Pécuchet (lecture mentionnée 30 septembre-23 octobre 1872, Carnet 15, fo 64, p. 509). Ces notes sur le Cours d'esthétique ont été publiées par G. Séginger, Gustave Flaubert 5, Minard, 2005, p. 247-330. 
L'homme à la soutane s'assit près d'eux ; - et Pécuchet aborda le christianisme.

- «Aucune religion n'a établi aussi bien cette vérité :

"La Nature n'est qu'un moment de l'idée!" »

- «Un moment de l'idée ?» murmura le prêtre, stupéfait.

- «Mais oui ! Dieu, en prenant une enveloppe visible, a montré son union consubstantielle avec elle.»

- «Avec la Nature? Oh! oh!»

- «Par son décès, il a rendu témoignage à l'essence de la mort, donc, la mort était en lui, faisait, fait partie de Dieu.» ? L'ecclésiastique se renfrogna. «Pas de blasphèmes! c'était pour le salut du genre humain qu'il a enduré les souffrances...» - «Erreur ! On considère la mort dans l'individu, où elle est un mal sans doute, mais relativement aux choses, c'est différent. Ne séparez pas l'esprit de la matière ! »

- «Cependant, monsieur, avant la Création...»

- «Il n'y a pas eu de création. Elle a toujours existé. Autrement ce serait un être nouveau s'ajoutant à la pensée divine, ce qui est absurde.»

Le prêtre se leva. Des affaires l'appelaient ailleurs. (g 224, fo 177$)^{21}$

Philosophie «Liebig» peut-être, mais Flaubert donne à ces concentrés une étonnante vertu polémique, en jouant d'une sorte de mimétisme textuel, très proche de ce qui est rapporté, cité, résumé, et dans une aura d'inintelligibilité. Intertextualité et interdiscursivité atteignent ainsi une force polémique particulièrement efficace.

C'était le cas aussi (le livre joue de symétries constitutives) avec Bouvard exposant Spinoza (Pécuchet est du côté d'un «idéalisme», d'où son adoption de l'idéalisme hégélien, Bouvard est du côté d'un «rationalisme » et d'un «monisme», d'où son adoption de Spinoza) :

Bouvard imagina que Spinoza peut-être, lui fournirait des arguments, et il écrivit à Dumouchel, pour avoir la traduction de Saisset 22 .

[...]

Ainsi notre monde n'est qu'un point dans l'ensemble des choses - et l'univers impénétrable à notre connaissance, une portion d'une infinité d'univers émettant près du nôtre des modifications infinies. L'Étendue enveloppe notre univers, mais est enveloppée par Dieu, qui contient dans sa pensée tous les univers possibles, et sa pensée elle-même est enveloppée dans sa Substance.

De l'Étendue à la Substance, cette dernière phrase construit un chiasme philosophique particulièrement efficace, comme un concentré de Spinoza, qui semble porter à la limite du pensable 23 :

Il leur semblait être en ballon, la nuit, par un froid glacial, emportés d'une course sans fin, vers un abîme sans fond ${ }^{24}$, - et sans rien autour d'eux que l'Insaisissable, l'Immobile, l'Éternel. C'était trop fort. Ils y renoncèrent. (g 224, fo $168-169)^{25}$

Comme dans les autres expériences où le seuil de difficulté à comprendre est atteint par les bonshommes, et dans les moments de détresse intellectuelle, le secours final est dans le passage à «plus simple» : «Et désirant quelque chose de moins rude, ils achetèrent le Cours de philosophie, à l'usage des classes, par Monsieur Guesnier», référence fictive, intertextualité de commodité.

\section{Fiction}

«Donner comme vraies des indications bibliographiques fausses » écrit Flaubert sur le premier scénario général du roman (gg 10, fo 5). On trouve des références

21. GF, p. 301-302; LP, p. 315.

22. Flaubert imagine dans les brouillons plusieurs voies pour le passage à Spinoza : «Descartes par la définition de la substance et de la négation de la création les mène à Spinosa » $\left(\mathrm{g} 225^{7}\right.$, fo $\left.854 \mathrm{v}^{\circ}\right)$; «Spinosa - panthéisme = identité de la substance » (g 2257, fo 870 vo); «La négation des Causes finales les mène au cœur du système de Spinosa » (g 2257, fo 776 vo). La «synthèse» que donne Flaubert (ils «comprirent ceci :») reprend presque textuellement plusieurs des notes qu'il a relevées dans l'Introduction critique aux euvres de Spinoza de Saisset (Charpentier, 1860), accompagnant sa traduction de Spinoza (1 re éd. en 2 vol. 1842, 2e éd. avec le Tractatus, 1861). Ces notes (g 2266 , fo 37-38 vo) ont été publiées par A. Yamazaki, voir note 20. L'Introduction de Saisset est à la fois une présentation et une critique de Spinoza, pour écarter du spiritualisme cousinien toute suspicion de «panthéisme».

23. Saisset écrivait, plus analytiquement : «L'idée de l'étendue enveloppe notre univers; mais elle-même est enveloppée par l'idée de Dieu, qui contient tous les univers possibles. Et Dieu enfin enveloppe ce nombre innombrable d'univers dans sa Pensée, et sa Pensée elle-même dans sa Substance, dernier fond qui contient tout...» (Introduction critique..., op. cit., p. 91).

24. Saisset évoque le Dieu de Spinoza comme «profondeur insondable que la pensée humaine adore en s'y abîmant» (p. 91). Flaubert avait développé un tel moment «spinoziste»d'appréhension de l'infini en expérience sensible d'ascension vertigineuse dans La Tentation de saint Antoine de 1874 , VIe partie.

25. GF, p. 289-290; LP, p. 302-303. 
bibliographiques fictives réparties dans la plupart des chapitres du roman. Le premier chapitre fait mention d'un Manuel du magnétiseur, dans la bibliothèque de Pécuchet, qui annonce le Guide du magnétiseur de Montacabère, utilisé au chapitre VIII. Flaubert joue avec l'érudition, comme plus tard Borges, ou Georges Perec dans Un cabinet d'amateur, ou encore Pierre Michon dans Les Onze. Il attribue probablement fictivement à «Christophe» la citation de l'article «Dessin, art du» du Dictionnaire des idées reçues : "Se compose de trois choses. "La ligne, le grain et le grainé fin, de plus, le trait de force. - Mais le trait de force! il n'y a que le Maître seul qui le donne" (Christophe) ${ }^{26}$. » La même citation figure au chapitre X sans nom d'auteur ${ }^{27}$, mais dans un brouillon elle est attribuée aux «Principes et éléments de Courbeval» (g 2259, fo 1148) puis, sur le manuscrit autographe, «le manuel de Courteval » est biffé (g 22510, fo 226) : l'une et l'autre références semblent inventées. Au chapitre V, de la littérature, Flaubert attribue à l'auteur Auguste Ricard un titre de roman, Le Porteur d'eau, qui n'est pas attesté dans les bibliographies, mais qui pastiche des titres de romans et des vaudevilles des années 1820 .

Dès les premières notes scénariques pour Bouvard et Pécuchet, datées de 1863, Flaubert envisage aussi pour la Copie d' "insérer là tout ce que l'on veut comme contrastes de faits, pastiches de style. / Mettre des morceaux vrais et des morceaux typiques. » (Carnet 19, fo 40 vo28.) Les dossiers comprennent en effet des pastiches de lettres, de poèmes, ou de prose, qui alternent avec des «morceaux vrais » 29 . Dans un scénario pour la Copie intitulé «Morceaux pastiches», Flaubert donne des exemples de morceaux «typiques» : «- article ultraradical, /- article ultraconservateur, /- article genre Vie parisienne [...]», et détaille les «Morceaux poétiques trouvés dans les papiers du clerc» (gg 10, fo 68). Flaubert prévoyait aussi d'inclure, parmi d'autres «morceaux inventés», la lettre canular de «Marengo l'Hirondelle» que lui adresse George Sand, le 4 décembre $1866^{30}$. Le pastiche et la farce, dans la tradition des farces du Garçon, est un plaisir littéraire que Flaubert partage avec ses amis. Avec Du Camp et Bouilhet, il rédige dans les années 1840 un pastiche inachevé de tragédie La Découverte de la vaccine, dont quelques fragments sans nom d'auteur figurent sous la catégorie des «périphrases » 31 . La pochade écrite en 1863 et 1867 , Vie et travaux du R. P. Cruchard ${ }^{32}$, comprend quelques lignes d'une bibliographie imaginée des travaux de Cruchard, un des surnoms que se donne Flaubert.

Dans la plupart des chapitres du roman, toutefois, l'intertextualité fictive dépasse la simple vocation ludique. Les références fictives désignant un nom d'auteur et un titre imaginaires ont une fonction épistémologique dans le roman encyclopédique. Ce sont des synthèses de théories et de débats d'époques que Flaubert construit à partir de ses notes de lecture. Ainsi, au chapitre III, le feuilleton inventé «De l'enseignement de la géologie » démonte à partir de la modernité de la géologie, inspirée des travaux de Lyell, la théorie du déluge de Cuvier ${ }^{33}$. La Mnémotechnie de Dumouchel (chapitre IV) combine explicitement «les trois systèmes d'Allevy, de Paris, et de Feinaigle»; L'Examen du socialisme par Morant (chapitre VI) condense les notes de Flaubert sur le «socialisme»; le Cours de philosophie de M. Guesnier construit un montage ironique de l'éclectisme (chapitre VIII). L'Examen du Christianisme par Louis Hervieu (chapitre IX) recouvre à la fois la pensée antireligieuse du XVIII' siècle et l'exégèse du christianisme que développe le comparatisme religieux du XIXe siècle 34 . Sous le nom d'Hervieu, Flaubert reprend tour à tour des arguments

26. La mention de l'auteur est ajoutée et l'ensemble est biffé.

27. GF, p. 366-367; LP, p. 384.

28. Voir Gustave Flaubert, Carnets de travail, op. cit., p. 300.

29. Une note scénarique pour la Copie comprend un plan de poème «préhistorique» (g 2262 , fo 14 ) ; on trouve également un pastiche de «style troubadour», accompagné de la mention «Trouver un titre et un nom» (g 2267, fo 12), et un pastiche de lettre forgé par Jules Duplan (g 2262, fo 292). Sur ce point et sur les «forgeries bibliographiques » de Flaubert, voir Stéphanie Dord-Crouslé, «"Donner comme vraies des indications bibliographiques fausses" : construction concertée et glissements contrôlés dans Bouvard et Pécuchet », Revue Flaubert, no 15, 2017 (en ligne sur flaubert.univ-rouen.fr).

30. L'autographe est reproduit dans l'édition du Centenaire et dans la Correspondance (en ligne sur flaubert.univ-rouen.fr).

31. Voir g 2263 , fo 8-9. Les morceaux font suite à deux extraits du poème de Delavigne du même titre.

32. Vie et travaux du R. P. Cruchard, et autres inédits, M. Desportes et Y. Leclerc (éd.), Publications des universités de Rouen et du Havre, 2005. 33. Voir à ce sujet Jiwoon Jung, «La géologie en feuilleton. La genèse de l'enseignement de la géologie », Flaubert. Revue critique et génétique, en ligne le 12 mai 2019 sur openedition.org.

34. Voir à ce sujet Anne Herschberg Pierrot, «Bouvard et Pécuchet et la critique rationaliste», dans Voir, croire, savoir. Les épistémologies de la création chez Flaubert, P.-M. de Biasi, A. Herschberg Pierrot, B. Vinken (dir.), De Gruyter, 2015, p. 201-219. 
critiques relevés chez divers auteurs rationalistes. Les notes de lecture, puis les notes de synthèse (les «notes de notes ») et les brouillons permettent de suivre la condensation des argumentaires dans le dialogue. Le manuel est invoqué dans la discussion sur le dogme, à propos de l'enfer, du péché originel et de la Trinité. L'Ancienne et la nouvelle foi, de David Friedrich Strauss (1876), et surtout l'Examen critique des doctrines de la religion chrétienne de Patrice Larroque (1860) sont les appuis dont Flaubert se sert pour construire les répliques de Bouvard. Mais Flaubert construit également les arguments du curé à partir de citations d'auteurs catholiques réfutés par Larroque. Son dialogue avec Bouvard est ainsi mené à partir d'auteurs rationalistes. Ensuite, Bouvard «ayant repassé son Louis Hervieu» (g 224, fo 20535), dans la discussion sur les miracles, c'est le Tractatus de Spinoza qui est mobilisé. Hervieu est encore invoqué lorsque Bouvard cherche à convaincre Pécuchet qui «avait espéré l'accord de la Foi et de la Raison» :

Bouvard lui fit lire ce passage de Louis Hervieu :

«Pour connaitre l'abîme qui les sépare, opposez leurs axiomes : «La Raison vous dit : le tout enferme la partie, et la Foi vous répond par la substantiation. Jésus communiant avec ses apôtres, avait son corps dans sa main, et sa tête dans sa bouche. «La Raison vous dit: On n'est pas responsable du crime des autres - et la Foi vous répond par le Péché originel.

«La Raison vous dit : trois c'est trois - et la Foi déclare que : trois c'est un.» (g 224, fo 20736)

L'extrait d'Hervieu entre guillemets est une citation presque littérale du livre de Pierre Lanfrey, référencé dans les scénarios, L'Église et les philosophes au XVIIIe siècle (1855, relu en 1879) ${ }^{37}$.

Une autre référence fictive, citée entre guillemets, mérite d'être signalée : il s'agit de l'ami de Dumouchel, «professeur de philosophie». Consulté une première fois au chapitre V pour l'esthétique («Un ami de Dumouchel, professeur de philosophie, leur envoya une liste d'ouvrages sur la matière. Ils travaillaient à part, et se communiquaient leurs réflexions $\left.{ }^{38} »\right)$, il est de nouveau consulté au moment de la politique, sur la question du droit divin :

L'ami de Dumouchel, ce professeur qui les avait éclairés sur l'esthétique, répondit à leur question dans une lettre savante. «La théorie du droit divin a été formulée sous Charles II par l'Anglais Filmer.

«La voici :
«Le Créateur donna au premier homme la souveraineté du monde. Elle fut transmise à ses descendants - et la puissance du Roi émane de Dieu. "Il est son image" écrit Bossuet. L'empire paternel accoutume à la domination d'un seul. On a fait les rois d'après le modèle des pères.

«Locke réfuta cette doctrine.» (g 224, fo 134-13539)

Le 24 juillet 1878, Flaubert avait demandé à Taine des renseignements sur la question du suffrage universel et du droit divin. Celui-ci lui répond le lendemain. La lettre fictive de l'ami de Dumouchel ne reprend pas directement la lettre de Taine, mais projette dans la fiction les indications de sa réponse. Flaubert écrit la réponse de l'ami de Dumouchel à partir des notes de lecture qu'il a prises, à la suite des conseils bibliographiques de Taine ${ }^{40}$ sur Bossuet, Filmer, Locke...

Ces jeux avec les noms fictifs jouent un rôle fondamental dans Bouvard et Pécuchet. Si l'intertextualité fictive a un rôle ludique jubilatoire, elle contribue à la composition des synthèses critiques qui mettent en perspective historique de manière très informée les controverses des années 18301860, période des dix premiers chapitres du roman à partir du temps de l'écriture - les années 1870-1880.

La consultation des notes et des brouillons permet aussi de suivre une intertextualité génétique qui reste silencieuse dans le texte, qu'elle soit dissimulée derrière les noms fictifs, ou laissée dans la genèse. Les travaux de Figuier sur le magnétisme sont absorbés dans le Manuel du magnétiseur de Montacabère (au chapitre VIII), ceux de Larroque dans le chapitre de la religion. Au chapitre de l'histoire (IV), le nom de Michelet, qui apparaît dans les scénarios et les brouillons comme un exemple de l'histoire partiale, est effacé au fil des brouillons : il reste hors des débats critiques ${ }^{41}$.

\footnotetext{
35. GF, p. 342 ; LP, p. 357.
}

36. GF, p. 343-344; LP, p. 359-360.

37. Voir Anne Herschberg Pierrot, art. cit., p. 210.

38. Ms g 224, fo 114 ; GF, p. 209 ; LP, p. 208.

39. GF, p. 241-242; LP, p. 248-249.

40. Antoine Compagnon, le premier, a proposé l'hypothèse de l'identification à Taine de l'ami de Dumouchel, dans La Troisième République des lettres, Seuil, 1983, p. 294-295.

41. Pour éviter certains anachronismes en fait, mais aussi, comme l'a indiqué Claude Duchet, parce que Michelet était «trop encombrant pour le projet de Flaubert» («Écriture et désécriture de l'histoire dans Bouvard et Pécuchet», Flaubert à l'œuvre, Flammarion, 1980, p. 128). 


\section{Trafic et contrebande}

\section{Intertextualité du récit}

Sans la consultation des notes de lecture, des scénarios et des brouillons, les références intertextuelles sont souvent difficiles et parfois impossibles à déceler. Flaubert fait un usage subtil des citations cachées et montrées, et des auteurs dissimulés ou exhibés dans le récit. Ainsi, dans la phase religieuse, Pécuchet s'essaie à la chasteté :

Une autre vertu lui manquait : la chasteté - car intérieurement, il regrettait Mélie, et le pastel de la dame en robe Louis XV, le gênait avec son décolletage.

Il l'enferma dans une armoire, redoubla de pudeur jusques à craindre de porter ses regards sur lui-même, et couchait avec un caleçon.

Tant de soins autour de la Luxure la développèrent. (g 224, fo 187$)^{42}$

Pécuchet, de fait, applique les recommandations du Manuel du séminariste, de l'abbé Louis Tronson. Dans ses notes sur l'abbé Tronson, Flaubert relève :

Le grand moyen pour prévenir bien des inconvénients contre la modestie qui pourraient arriver la nuit c'est de ne coucher jamais sans caleçon, c'est ainsi qu'en a jugé Saint Fulgence ${ }^{43}$.

Le Manuel du séminariste est cité deux folios plus loin :

- «Pourquoi gémis-tu pendant le repas?»

- « Nous devons manger en gémissant» répondit Pécuchet,

«car l'Homme par cette voie a perdu son innocence» phrase qu'il avait lue dans le Manuel du séminariste, deux volumes in-12 empruntés à M. Jeufroy. (g 224, fo 189)44

Ce n'est pas la phrase exacte de Tronson, mais approximativement celle que condense Flaubert dans ses notes : «Nous devons manger avec gémissement, considérant que c'est par cette voie que l'homme a perdu l'innocence et qu'il a pris occasion de se révolter mille fois contre son Dieu ${ }^{45}$.» Sauf que la formulation de Bouvard et Pécuchet opère un glissement de la motivation générale, de l'ordre de l'hypothèse («considérant que c'est par cette voie...»), à l'actualisation en une vérité factuelle («car l'Homme par cette voie a perdu son innocence »). Comme précédemment pour la «condensation » dans l'épisode de la chimie, il s'agit de passer d'une opinion, d'une idée, d'un dogme, présentés à titre d'hypothèse, à une affirmation de réalité.

\section{Tronquer, empiler}

La manipulation intertextuelle la plus fréquente, dans le premier, mais surtout dans le second volume, consiste à couper de leur contexte d'origine les citations (littérales ou trafiquées), et à empiler et effacer la chaîne des intertextes.

Parmi un ensemble de citations en faveur de l'esclavage, figure, après Bossuet, celle-ci :

Le trafic des esclaves n'est opposé ni à l'humanité, ni à la religion, ni à l'équité naturelle.

Mgr Bouvier, évêque du Mans, Institutiones theologiae,

chap. II, art. I, § 3, tome VI (g 2266, fo 182).

Cette phrase est extraite de La Morale de l'Église et la Morale naturelle (1866), de Marc-Lucien Boutteville, un auteur classé à "gauche», collaborateur de Proudhon, que Flaubert a déjà lu pour La Tentation de saint Antoine. Boutteville cite lui-même De l'esclavage chez les nations chrétiennes (Leipsick, A. Durr, 1860, p. 31-34) de Patrice Larroque, qui écrit de Mgr Bouvier :

L'auteur conclut nettement que la religion chrétienne, qui a trouvé l'esclavage établi partout, ne l'a pas défendu. Parlant ensuite de la traite des nègres, il la déclare permise, à condition que les nègres seront justement privés de leur liberté, qu'on les traitera humainement et qu'il n'y aura pas de fraude sur le marché. Ces conditions posées, il prétend que ce trafic n'est opposé ni à l'humanité, ni à la religion, ni à l'équité naturelle.

Le propos est engagé, et modalise la valeur de vérité des déclarations de Mrr Bouvier. La citation pour la Copie est donc une citation de citation, dont le contexte est passé sous silence. L'extrait, comme les citations des pères de l'Église ou de la Bible qui le précèdent, est trouvé chez

42. GF, p. 314 ; LP, p. 329.

43. Notes sur le Manuel du séminariste, dans Euvres complètes, Migne, t. I, 1857, p. 213, Entretien XIX, «Sur le coucher» (2266, fo 215 vo, notes reprises du Carnet 6, fo 22-24, Carnets de travail, p. 907-908). 44. GF, p. 317 ; LP, p. 332.

45. Notes, g 2266, fo 215 vo et Carnet 6, fo 24, p. 907. Tronson écrit : «la pénitence, qui nous doit faire manger avec gémissement, considérant que c'est par cette voie que l'homme a perdu son innocence, et qu'il lui a donné l'occasion de se révolter mille fois contre son Dieu», p. 174. 
Fig. 2 : Page de «Copie» pour le «Second volume», de la main d'Edmond Laporte, annotée par Flaubert : «Philosophie Causes finales». Ms g 226 ${ }^{1}$, fo 105.

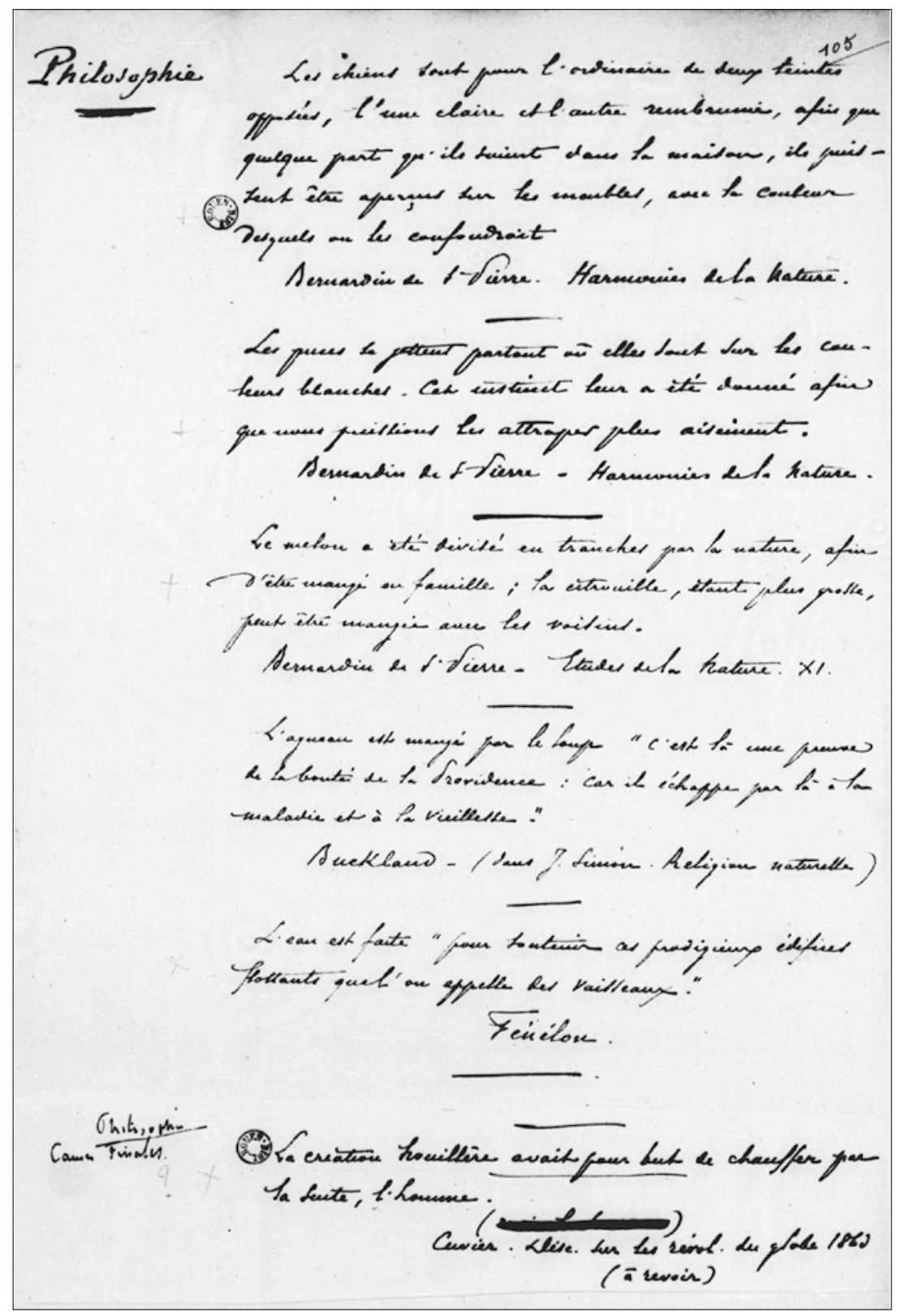

un auteur résolument opposé à l'esclavage46. On retrouve pour la Copie le procédé utilisé dans le premier volume, qui consiste à citer d'après des discours de réfutation.

Le nom de Boutteville émerge à la suite de l'extrait de Mgr Bouvier, signant une citation qui est en fait le résumé d'une page entière sur Bonaparte (p. 330). Laporte copie l'extrait condensé par Flaubert 47 :

Bonaparte rétablit l'esclavage et la traite par un décret du 30 Floréal an X (19 mai 1802) - vingt-quatre jours après le rétablissement du culte catholique.

Boutteville, p. 330. (g 2266, fo 182)
Cet ensemble de citations sur l'esclavage est en lien direct avec la discussion au «château» (chapitre IX) sur le christianisme et l'esclavage, entre le comte de Faverges et Bouvard :

46. Flaubert a relevé dans ses notes sur Boutteville une liste d'auteurs à citer («Esclavage v. les textes p. 305 \& sq.» (g 2266, fo 304 vo) que Laporte copie à la suite en g 2266, fo 182 . Voir à ce sujet Anne Herschberg Pierrot, «Le christianisme et l'esclavage», Revue Flaubert, no 13, 2013, en ligne sur flaubert.univ-rouen.fr.

47. À ceci près : Flaubert écrit «quarante-deux jours », que Laporte inverse en « vingt-quatre». 
Le comte croyant voir dans cette répartie une atteinte à la Religion l'exalta. Elle avait affranchi les esclaves.

Bouvard fit des citations, prouvant le contraire :

- Saint Paul leur recommande d'obéir aux maîtres comme à Jésus. - Saint Ambroise nomme la servitude un don de Dieu. - Le Lévitique, l'Exode et les Conciles l'ont sanctionnée. Bossuet la classe parmi le droit des gens. - Et Mgr Bouvier l'approuve. (g 224, fo 21248)

À la thèse du comte, Bouvard oppose un argumentaire de citations notées pour la Copie. Les extraits, montés à la suite, forment un réquisitoire contre les positions de l'Église et de ses défenseurs les plus intransigeants : preuves à l'appui, le dossier montre les contradictions entre les positions, réfute la pensée dogmatique en la confrontant à son histoire. Le montage des citations montre également l'historicité de la controverse, qui se développe au XIXe siècle à partir d'un thème diffusé par Chateaubriand. L'extrait du Génie du christianisme apparaît à la suite de trois pages qui disent le contraire :

... il est certain que personne n'a élevé la voix avec autant de courage et de force en faveur des esclaves, des petits et des pauvres, que les écrivains ecclésiastiques.

Chateaubriand, Génie du Christianisme, p. 60, t. IV. (g 2266, fo 185)

Les deux volumes sont donc extrêmement intriqués : à la condensation des dialogues répond le montage des exempla que forment les extraits de textes pour la Copie, des fragments d'opinions. Le roman construit un réseau de circulation entre les deux volumes, qui mobilise une participation active de la mémoire et du jugement du lecteur.

Un autre exemple de cette intertextualité spécifique entre les deux volumes est celui des «causes finales », sujet que Bouvard et Pécuchet rencontrent dans leur interrogation sur la nature, puis dans la phase philosophique. Au chapitre III, ils lisent les Harmonies de la nature de Bernardin de Saint-Pierre :

Ils s'étonnaient que les poissons eussent des nageoires, les oiseaux des ailes, les semences une enveloppe - pleins de cette philosophie qui découvre dans la Nature des intentions vertueuses et la considère comme une espèce de saint Vincent de Paul, toujours occupé à répandre des bienfaits ! (g 224, fo 63) 49

Les brouillons sont plus explicites. Flaubert écrit : «voyant partout dans l'univers des causes finales, des intentions vertueuses » (g 2252, fo 345), évoquant aussi «cette philosophie douceâtre qui aperçoit dans la Nature des intentions vertueuses » (g 2252, fo 348). Dans le second volume, sous la rubrique «Philosophie», Laporte reprend des extraits en série de Bernardin de Saint-Pierre, comme cette phrase souvent citée :

Le melon a été divisé par tranches par la nature, afin d'être mangé en famille; la citrouille, étant plus grosse, peut être mangée avec les voisins.

Bernardin de Saint-Pierre, Études de la nature, XI. (ms g 2261, fo 105, voir fig. 2)

ou :

Les chiens sont pour l'ordinaire de deux teintes opposées, l'une claire et l'autre rembrunie, afin que quelque part qu'ils soient dans la maison, ils puissent être aperçus sur les meubles, avec la couleur desquels on les confondrait.

Bernardin de Saint-Pierre, Harmonies de la nature.

Il cite aussi Buckland :

L'agneau est mangé par le loup «c'est là une preuve de la bonté de la Providence : car il échappe par là à la maladie de la vieillesse».

Buckland (dans J. Simon, Religion naturelle). (g 2261, fo 105)

Flaubert a relevé tous ces exemples dans le livre de Paul Janet, Les Causes finales (Germer Baillière, 1876). L'empilement intertextuel est effacé au profit de la citation brute - brute, mais sertie dans un contexte qui la tire de l'intertexte originaire et l'inscrit dans une série, en parallèle avec d'autres. La citation, simple, de Bernardin de SaintPierre, ou citée par la médiation d'un intertexte nommé (Buckland, dans J. Simon) est effectuée d'après un intertexte effacé (Paul Janet). Flaubert avait indiqué dans ses notes sur Paul Janet : «exemples de causes finales - sottes à copier p. 267, p. 270-271» (g 2266, fo 24 vo). Janet traite lui aussi ces exemples d' "applications puériles et frivoles des causes finales [...]. Quelques-unes de ces applications sont tellement ridicules, qu'on pourrait les croire inventées pour ridiculiser la théorie elle-même» (p. 267-268).

Cette intertextualité emboîtée se lit dans les listes pour la Copie, qui s'apparentent à des formes de non-sens et

48. GF, p. 350 ; LP, p. 367.

49. GF, p. 131 ; LP, p. 124. 
d'humour noir. Elles transforment un discours suivi en énumérations verticales : que ce soit le catalogue des meurtres enchaînés des rois de la Bible, retenu dans le Dictionnaire philosophique de Voltaire, ou, les contradictions entre auteurs sur le nombre de races humaines, énuméré dans La Descendance de l'homme de Darwin et retenu sous les catégories : «Énumérations » et «Contradictions de la science («Ethnographie» est biffé) :

Races humaines. Il y en a

2. Virey
3. Jacquinot
4. Kant
5. Blumenbach
6. Buffon
7. Hunter
8. Agassiz
11. Pickering
15. Bory Saint-Vincent
16. Desmoulins
22. Morton
60. Crawfurd
63. Burke.

243. (g 2261, fo 287)

La mise en liste radicalise le propos de Darwin, qui écrit dans La Descendance de l'homme:

L'homme a été étudié avec plus de soin qu'aucun autre être organisé, et cependant il y a entre des juges capables la plus grande divergence sur le fait, s'il faut le regarder comme une seule espèce ou race, ou deux (Virey), trois (Jacquinot); quatre (Kant); cinq (Blumenbach); six (Buffon); sept (Hunter); huit (Agassiz); onze (Pickering); quinze (Bory Saint-Vincent); seize (Desmoulins) ; vingt-deux (Crawfurd); ou soixante-trois d'après Burke.

Traduit de l'anglais par J. J. Moulinié, C. Reinwald, 1872 , t. I, p. 245.

Darwin renvoie lui-même en note à deux auteurs auxquels il reprend ces chiffres : il se crée ainsi un effet de mise en abîme de l'intertextualité scientifique, que rien ne distingue plus de la redite d'idées reçues.

Dans le même genre, toujours sous «Contradictions de la science», figure cette liste relevée du Traité d'hygiène de Becquerel :
Durée de la destruction complète des cadavres :

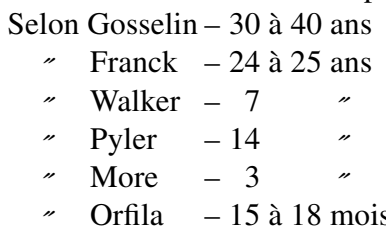

En France la moyenne admise est de 5 ans.

A. Becquerel, Traité élémentaire d'hygiène publique et privée, t. II. p. 261. (g 2264, fo 53)

\section{Fabriquer le péremptoire}

L'absence de commentaire laisse le lecteur seul devant l'affirmation définitive. Faire entendre le péremptoire, au besoin le fabriquer, telle est la ressource principale contre l'arrogance. Sous la rubrique «Beautés du peuple», on lit ainsi :

Les malades répondent si bêtement que pour le diagnostic de beaucoup de maladies l'exploration muette est préférable aux données fournies par l'interrogation des malades. Jean Bouillaud, Essais sur la philosophie médicale.

(g 2261, fo 95)

Cette formulation est un raccourci relevé des notes de lecture de Flaubert, qui perçoit le comique de l'arrogance médicale dans le texte de Bouillaud (g 2267, fo 84). Celui-ci écrit :

Il est des malades qui savent si peu s'observer, dont l'intelligence naturelle est si obtuse, qu'on ne peut en obtenir aucune réponse satisfaisante. [...] Certes, nous ne méprisons pas, encore une fois, les données, les renseignements que nous nous procurons au moyen de l'interrogation des malades, et nous avouons volontiers par conséquent que la médecine vétérinaire possède pour ainsi dire un sens de moins que la médecine humaine. Ce que nous avons voulu dire uniquement, c'est que, pour le diagnostic de beaucoup de maladies, si nous avions à choisir entre les données fournies par l'exploration muette, en quelque sorte, et celles fournies par l'interrogation des malades, nous devrions assurément préférer les premières. Essais sur la philosophie médicale,

De Just Rouvier et E. Le Bouvier, 1836, p. 139-140.

En classant l'extrait sous «Beautés du peuple», Flaubert fait du jugement de bêtise (celle du peuple, celle du médecin) l'objet même de l'ironie. 
Un autre exemple de l'effet de péremptoire lié au raccourci génétique de Flaubert est cet extrait de l'Histoire de la Révolution de 1848, de Daniel Stern. L'auteur relève les déclarations enthousiastes d'adhésion du clergé catholique à la République après le 24 février 1848 : celles de Mgr Affre, de Lacordaire, de L'Univers. Flaubert retient sous «Flagorneries envers le peuple»:

Après le 24 févr[ier] Lacordaire prend pour texte à SaintMerry : «ce peuple superbe en sa colère»

L'Univers se déclare Républicain. (g 2261, fo 204)

Les deux phrases proviennent de ses notes de lecture, qui reprennent la formule de Lacordaire mais résument en une phrase la longue déclaration de L'Univers, «journal du parti catholique», citée par D. Stern (Histoire de la Révolution de 1848, Charpentier, 1862, t. I, p. 394-395).

Un degré de plus dans le geste de manipulation consiste non plus à citer un texte mais à lui substituer un commentaire. «Voltaire n'avait pas lu Bacon!» : cette phrase sur Voltaire, classée dans la rubrique «Grands hommes » n'est pas une citation de Joseph de Maistre, mais l'exclamation de Flaubert à la lecture de ces lignes : «On voit ici une nouvelle fois la preuve que Voltaire, ainsi que la plupart des panégyristes de Bacon, ne l'avait pas lu. » (Joseph de Maistre, Examen de la philosophie de Bacon, Paris, Poussielgue-Rusand, Lyon, Pélagaud, Lesne et Crozet, t. II, 1836 , p. 356 , g $226^{6}$, fo 271 vo.)

\section{Parallèles et contradictions : construire l'intertextualité entre les citations}

À côté de l'empilement intertextuel, qui conduit à des raccourcis et des citations privées de leur contexte d'origine, il est une autre forme de relation intertextuelle, qui est horizontale et circulaire. Elle procède des relations établies entre les extraits par montage sur une même page, ou par renvoi d'un auteur à l'autre. La circulation intertextuelle est alors posée par la construction du texte et en appelle à effectuer, par la lecture, les rapprochements établis entre les opinions des auteurs mis en contradiction ou en parallèle.

Sur Napoléon, par exemple, Flaubert s'amuse à faire dialoguer deux jugements contradictoires de Lamennais, à vingt ans de distance, avec l'extrait de Joseph de Maistre (g 2263, fo 35), qu'il ajoute entre eux deux :
Napoléon était ennemi par instinct de la civilisation.

Lamennais, De la Religion dans ses rapports avec l'ordre politique [Liberté d'enseignement, 1814].

Napoléon le moderne Attila, moins civilisé que le premier.

De Maistre, Du Pape, Liv. 3, ch. Ier.

Napoléon est le plus grand homme des temps modernes. Lamennais, Affaires de Rome.

Flaubert rapproche aussi sur une page des définitions du poète, toutes étrangères à l'art, de Renan, Augier mais aussi de Stendhal, Addison, Damiron et Béranger (g 22633, fo 59) :

Esthétique

Poète

Mettre avant la théorie de Michelet sur la joie de l'inventeur

Il faut pour les arts des gens un peu mélancoliques et malheureux.

Stendhal, Promenades dans Rome, tome II, p. 440.

Esthétique Comment pourrait-il être poète? Il n'a jamais vu de montagnes!

Pour être poète Mot d'Addison à rapprocher de Proudhon qui blâme les voyages.

Définition du Poète, à ajouter à toutes les autres

Qu'un homme ému de la beauté du devoir s'efforce de faire passer son impression dans ses actions, que selon les circonstances, il soit brave, désintéressé, juste, humain ou religieux, et qu'il le soit excellemment, il est poète alors non par le génie il est vrai, mais par le cœur et le caractère

Damiron, Cours de Philosophie. Morale, Préface, p. 1.

Napoléon «le plus grand poète des temps modernes ».

Jésus-Christ «poète».

Béranger, Préface.

Renan.

«Ô poète». La femme d'un notaire à son mari. Augier, Gabrielle.

Les références par renvois («à rapprocher», «voyez»...) soulignent les contradictions et parallèles entre les affirmations. Le jugement sans appel du Dr Belouino sur les romans : «Ce sont les romans qui jettent à la prostitution le 
plus grand nombre de ses victimes » est démenti en marge : «Voy. Parent du Chatelet : les prostituées ne savent pas lire» (g 2263, fo 85) ${ }^{50}$. Elles rapprochent aussi les esthétiques d'auteurs idéologiquement éloignés, comme de Maistre, Nisard ou Gaume et Proudhon, rassemblés dans un commun mépris de l'art. Flaubert met ainsi en parallèle ce jugement de J. de Maistre :

(À propos de la Phèdre de Racine) Qu'est-ce que tout cela nous fait à nous, chrétiens ou athées du XIXe siècle? Rien n'est plus étranger à nos mœurs, à notre croyance, à notre philosophie même.

[Joseph] de Maistre, Exam. de la philos. de Bacon,

t. II, p. 298.

Et celui de Proudhon («Id. de Proudhon») (g 2263, fo 52) cité dans un autre dossier pour la Copie sous «Socialisme» :

Que me fait l'Art égyptien avec ses pyramides et ses symboles. Est-ce que ma conscience est égyptienne. Que me font les monuments de l'art grec lui-même!

Du Principe de l'Art et de sa destination sociale, 1865, p. 325. (g 2265, fo 273)

Proudhon est également relié à Nisard pour sa condamnation des voyages d'un artiste (g 2263, fo 57), et à l'abbé Gaume pour son dénigrement de la Renaissance (g 2263, fo 63).

\section{Intertextualité et genèse}

Les dossiers de Bouvard et Pécuchet mêlent étroitement intertextualité et genèse, dans un travail qui conduit à convertir sans cesse l'exogenèse en une endogenèse particulièrement complexe. Les notes de lecture absorbées dans les dix chapitres du roman refont surface sous forme d'extraits montrés pour la Copie. Et de cette origine génétique et intertextuelle commune résulte un texte qui établit une circulation intratextuelle entre les deux volumes de cet «ensemble» tant recherché par Flaubert. Un exemple encore le montre : celui des anecdotes édifiantes de Mme de Noaris au chapitre IX. Flaubert intègre d'abord aux brouillons de ce chapitre le récit d'anecdotes tirées des Discussions religieuses de l'abbé Daux (g 2259, fo 1125 vo). Le texte n'en présente plus qu'une mention, rapportée vaguement à «des opuscules », et souligne leur caractère de rengaine :
Le soir, un valet du château lui apporta une hottée d'opuscules, relatant des paroles pieuses du grand Napoléon, des bons mots de curé dans des auberges, des morts effrayantes advenues à des impies. Mme de Noaris savait tout cela par cœur, avec une infinité de miracles. (g 224, fo 20551)

Coupées des brouillons, où Flaubert les avait citées, les anecdotes des Discussions religieuses sont retenues pour la Copie sous la rubrique «Impies impunis » (g 2266, fo 192).

Cet exemple nous invite à considérer que l'intertextualité proprement littéraire s'inscrit dans une intertextualité plus large, discursive, religieuse, politique, scientifique, une interdiscursivité médiatisée cependant par des discours de vulgarisation, de pédagogie, par les articles de presse. Certains extraits sont même dépourvus de toute référence. Sous «Bizarreries», on peut lire :

La comédie doit-elle s'écrire en prose ou en vers?

La découverte de l'Amérique a-t-elle été utile ou funeste au genre humain?

Télémaque est-il un poème ou un roman? (g 2261, fo 286)

La première question fait écho à l'une des interrogations esthétiques de Bouvard et Pécuchet au chapitre de la littérature 52 , la seconde est un sujet de concours de l'Académie de Lyon, extrait de l'article «Raynal» de la Biographie Michaud, cité pour la Copie :

Raynal donna à l'Académie de Lyon un prix de douze cents francs, pour l'auteur du meilleur mémoire sur cette question : «La découverte de l'Amérique a-t-elle été utile ou nuisible au génie humain?» (g 2261', fo 281)

Le montage fait ressortir l'académisme des questions, et leur inanité. Cette interdiscursivité ne se limite pas à la reprise d'écrits transformés au fil des médiations. Elle relève aussi de l'oralité et du relais des conversations, qui moulinent les idées reçues - comme ces fragments de citations dans

50. Alexandre Parent du Châtelet, De la prostitution dans la ville de Paris, Paris, J.-B. Baillière, 1857. Flaubert relève cette contradiction dans le Carnet 15 , fo 27 vo, p. 489.

51. GF, p. 341 ; LP, p. 357.

52. «Une illumination lui vint : s'ils avaient tant de mal, c'est qu'ils ne savaient pas les règles. / Ils les étudièrent, dans La Pratique du théâtre par d'Aubignac, et dans quelques ouvrages moins démodés. / On y débat des questions importantes : si la comédie peut s'écrire en vers $[\ldots]$ » (g 224, fo 111), GF, p. 204-205; LP, p. 203-204. 
Le Dictionnaire des idées reçues : par exemple, la plaisanterie désuète de l'article «Banquet» : «Un farceur doit dire "au banquet de la vie, infortuné convive"», en référence à un poème de Gilbert 53 . L'intertextualité se donne sur le mode de l'allusion, mais une allusion qui n'ouvre que sur le ressassement de paroles gelées. Les dossiers pour la Copie se font ainsi l'écho répété des «scies» de Lamartine sur Rabelais, des rengaines sur Voltaire; des mots de Thiers, non référencés, repris pour la Copie ou le Dictionnaire des idées reçues (articles «Faute» et «Liberté», qui inscrivent une mémoire du second Empire). Certains écrits sont particulièrement ciblés, comme ceux de Joseph de Maistre. Il y va, dans le comique de l'absurde, et dans la farce, d'une irritation de Flaubert contre la bêtise de l'aplomb, et, d'une exaspération contre la «commune prétention à détenir ou être la vérité $54_{\gg}$ - comme il en va des dieux montrés et pulvérisés dans La Tentation de saint Antoine.

$*$

«Tout dogmatisme m'exaspère ${ }^{55}$. » La mise en œuvre de l'intertextualité et de l'interdiscursivité dans les deux volumes est une riposte ironique, puissante, au dogmatisme. Le montage des textes n'égalise pas les sottises dans le rire, ou dans la copie sans distance. Dans le montage des citations et des allusions, dans l'entrelacs des deux volumes, à travers la polyphonie des voix, le projet de l'auteur est bien le ravage des opinions péremptoires, des jugements d'autorité. Les gesticulations et les ratages de Bouvard et Pécuchet participent d'une œuvre de fiction écrite contre la haine de l'art et le mépris de la science, contre la puissance des bienpensants, et de l'ordre moral, celle d'une religion toujours étroitement impliquée dans le politique. En 1863, hésitant entre deux plans de roman moderne, ce qui sera L'Éducation sentimentale (celui que finalement il écrira d'abord) et ce qui sera plus tard Bouvard et Pécuchet, Flaubert confiait à Jules Duplan : «Quant au second dont j'aime l'ensemble j'ai peur de me faire lapider par les populations ou déporter par le gouvernement, sans compter que j'y vois des difficultés d'exécution, effroyables. » À la fin de 1879, presque au bout de son œuvre, il en retrace la visée et s'interroge : «J'écris à l'intention de quelques raffinés. Peut-être sera-ce une lourde sottise? À moins que ce ne soit quelque chose de très fort? Je n'en sais rien! » (à Gertrude Tennant, 16 décembre 1879).

53. Voir Gustave Flaubert, Le Dictionnaire des idées reçues, A. Herschberg Pierrot (éd.), Le Livre de Poche, 1997.

54. Jacques Neefs, «L'exposition littéraire des religions», dans Travail de Flaubert, G. Genette et T. Todorov (éd.), Seuil, 1983, p. 130.

55. À Edma Roger des Genettes, 13 mars 1879.

Anne Herschberg Pierrot, professeur émérite à l'université Paris 8, corédactrice de la revue en ligne Flaubert. Revue critique et génétique (en ligne sur openedition.org), a édité Le Dictionnaire des idées reçues au Livre de Poche et dirigé notamment Flaubert, l'empire de la bêtise (2012); avec Jacques Neefs, elle a publié Bouvard et Pécuchet. Archives et interprétation (Éditions Cécile Defaut, 2014) et prépare l'édition de La Tentation de saint Antoine et de Bouvard et Pécuchet au tome V des CEuvres complètes de Flaubert à la Pléiade (2021).

ahp2@orange.fr

JACQUES NEEFS, professeur émérite à Johns Hopkins University et à l'université Paris 8, membre de l'American Academy of Arts and Sciences, a notamment édité au Livre de Poche Madame Bovary (1999, nouvelle édition en 2019), et Salammbô (2011). Avec Anne Herschberg Pierrot, il a publié Bouvard et Pécuchet. Archives et interprétations (Éditions Cécile Defaut, 2014) et édite La Tentation de saint Antoine et Bouvard et Pécuchet au tome v des Euvres complètes de Flaubert à la Pléiade (2021). 


\section{L'intertextualité ravageuse de Bouvard et Pécuchet}

Avec Bouvard et Pécuchet Flaubert met en œuvre une relation particulièrement complexe à la bibliothèque, à l'intertextualité et l'exogenèse. Les livres ouverts par les deux encyclopédistes, lors des dix premiers chapitres du roman, devaient faire retour sous la forme d'extraits classés et copiés, dans un «second volume», avec le Dictionnaire des idées reçues et le Catalogue des idées chic. L'ensemble, indissociable pour Flaubert, visait à poser les limites de la pensée et du jugement, à pulvériser les discours d'autorité, à interroger le bien-fondé des savoirs et des disciplines, en mêlant le jeu et le sérieux et instaurant ce que Flaubert nomme le «comique d'idées». En nous appuyant sur les notes de lecture, les brouillons du texte et les extraits de la «Copie», nous montrons, par quelques exemples, comment Flaubert condense, manipule, détourne les énoncés de savoir, comment il joue de l'érudition, mais aussi comment par la circulation génétique et intratextuelle entre les volumes, il construit une œuvre inouïe où la fiction démonte la «commune prétention à détenir ou être la vérité ».

In Bouvard and Pécuchet, Flaubert dramatizes the particularly complex relation between the library, intertextuality, and exogenesis. The books opened by the two encyclopedists in the first ten chapters of the novel return in the form of organized, copied-out excerpts in the "second volume", that of the Dictionnaire des idées reçues and the Catalogue des idées chic. Together - the two being indissoluble for Flaubert - these books aim to sets the limits of thought and judgment, to shatter the discourses of authority, and to interrogate the legitimacy of domains of knowledge and disciplines, combining the playful with the serious and inaugurating what Flaubert calls the "comedy of ideas". Relying upon reading notes, textual drafts and excerpts from the "Copy", we will show, through several examples, how Flaubert condenses, manipulates and undermines pronouncements of knowledge, how he plays with erudition, but also how through genetic and intertextual circulation between the volumes, he constructs an unforgettable work in which fiction dismantles the "common pretension of knowing or being the truth".

Mit Bouvard und Pécuchet setzt Flaubert eine besonders komplexe Beziehung zur Bibliothek, Intertextualität und Exogenese um. Die von den beiden Enzyklopädisten in den ersten zehn Kapiteln des Romans aufgeschlagenen Bücher sollten in Form von klassifizierten und kopierten Auszügen in einem „,Zweiten Band“ zusammen mit dem Dictionnaire des idées reçues und dem Catalogue des idées chic zurückgegeben werden. Das Ganze, das für Flaubert untrennbar war, sollte die Grenzen des Denkens und Urteilens festlegen, Autoritätsreden pulverisieren, die Gültigkeit von Wissen und Disziplinen in Frage stellen, Spiel und Ernst vermischen und das etablieren, was Flaubert die „Komik der Ideen "nannte. Anhand von Lesevermerken, Textentwürfen und Auszügen aus der „Kopie“ zeigen wir anhand einiger Beispiele, wie Flaubert Wissensaussagen verdichtet, manipuliert und umlenkt, wie er mit der Gelehrsamkeit spielt, aber auch, wie er durch die genetische und intratextuelle Zirkulation zwischen den Bänden ein beispielloses Werk konstruiert, in dem die Fiktion den ,gemeinsamen Anspruch, die Wahrheit zu halten oder die Wahrheit zu sein" («commune prétention à détenir ou être la vérité») demontiert.
Con Bouvard et Pécuchet, Flaubert pone en práctica una relación particularmente compleja con la biblioteca, la intertextualidad y la exogénesis. Los libros abiertos por los dos enciclopedistas, durante los diez primeros capítulos de la novela, debían reaparecer en forma de fragmentos clasificados y copiados, en un "segundo volumen", con el Dictionnaire des idées reçues y el Catalogue des idées chic. Este conjunto, indisociable para Flaubert, tenía como objetivo establecer los límites del pensamiento y el juicio, pulverizar los discursos de autoridad, cuestionar la conformidad de los conocimientos y las disciplinas, mezclando el juego con la seriedad, e instaurando lo que Flaubert denomina la "comicidad de las ideas". Basándonos en las notas de lecturas, los borradores del texto y los fragmentos de la "Copia", tratamos de mostrar, a través de algunos ejemplos, de que manera Flaubert condensa, manipula, desvía los enunciados de conocimiento, como juega con la erudición, pero también como construye, a través de la circulación genética e intratextual entre los volúmenes, una obra insólita en la que la ficción desmonta la "pretensión común de poseer o ser la verdad".

Com Bouvard et Pécuchet Flaubert implementa uma relação particularmente complexa com a biblioteca, a intertextualidade e a exogénese. Os livros abertos pelos dois enciclopedistas, durante os primeiros dez capítulos do romance, deviam reaparecer sob a forma de excertos classificados e copiados, num "segundo volume", com o Dictionnaire des idées reçues e o Catalogue des idées chic. O conjunto, indissociável para Flaubert, visava estabelecer os limites do pensamento e do julgamento, pulverizar os discursos da autoridade, questionar a legitimidade do saber e das disciplinas, misturando o jocoso e o sério e instaurando o que Flaubert chama o "cómico de ideias". Com base nas notas de leitura, nos rascunhos do texto e nos excertos da "Copie", mostramos, através de alguns exemplos, como Flaubert condensa, manipula e contorna os enunciados do saber, como brinca com a erudição, mas também como, através da circulação genética e intratextual entre os volumes, constrói uma obra incrível onde a ficção desmonta a "comum pretensão de deter ou de ser a verdade".

In Bouvard e Pécuchet, Flaubert dà vita a una relazione particolarmente complessa con la biblioteca, l'intertestualità e l'esogenesi. I libri aperti dai due enciclopedisti durante i primi dieci capitoli del romanzo sarebbero dovuti ritornare, sottoforma di estratti ordinati e copiati, in un "secondo volume", congiuntamente al Dizionario dei luoghi comuni e al Catalogo delle idée chic. L'insieme - per Flaubert indissociabile - puntava a porre $\mathrm{i}$ limiti del pensiero e del giudizio, a polverizzare il discorso dell' autorità, a interrogare il fondamento dei saperi e delle discipline, mescolando gioco e serietà e instaurando ciò che Flaubert chiama "la comicità delle idee". Basandosi sulle note di lettura, gli abbozzi del testo e gli estratti della "Copia", l'articolo dimostra, tramite qualche esempio, in che modo Flaubert condensa, manipola e deforma gli enunciati del sapere, come si prende gioco dell'erudizione, ma anche in che modo costruisce, grazie alla circolazione genetica e intratestuale tra i volumi, un'opera senza precedenti in cui la finzione smonta la "pretesa comune di possedere o essere la verità". 